\title{
PROSPECTS FOR THE USE OF THE METHOD OF INTRAVESICAL HYPERTHERMIC CHEMOTHERAPY IN THE TREATMENT OF PATIENTS WITH NON-MUSCLE INVASIVE BLADDER CANCER
}

\section{Roman Chystiakov}

The aim: to compare disease-free survival time (DFS) in high-risk non-muscle-invasive bladder cancer patients treated with adjuvant hyperthermic intravesical chemotherapy and standard BCG therapy.

Materials and methods: patients were divided into 2 groups. Group 1 (control) included patients who received adjuvant therapy after TURB with BCG vaccine (BCG therapy group; $n=50$ ), group 2 (study group) included patients (HIVEC ${ }^{\circledR}$ therapy group; $\left.n=46\right)$, who received adjuvant intravesical chemotherapy using a Combat BRS HIVEC ${ }^{8}$ device for local hyperthermia.

Results: median follow-up was 23 months (range 4 - 36). Tumor recurrence was reported in 19 patients receiving intravesical BCG therapy and in 8 patients receiving intravesical hyperthermic chemotherapy. The incidence of DFS in patients receiving chemohyperthermy was statistically higher than in patients receiving BCG therapy (log-rank test result: $p=0.029)$.

Conclusions: The method of hyperthermic intravesical chemotherapy significantly increased the 2-year disease-free survival rate - $82.6 \%$ versus $62 \%$ BCG therapy group $(p=0.025)$. Its use in the future will allow increasing the frequency of organ-preserving treatment of patients with primary and recurrent muscular-non-invasive bladder tumors Keywords: Non-muscle invasive bladder cancer, hyperthermic intravesical chemotherapy, BCG therapy

How to Cite:

Chystiakov, R. (2021). Prospects for the use of the method of intravesical hyperthermic chemotherapy in the treatment of patients with non-muscle invasive bladder cancer. ScienceRise: Medical Science, 2 (41), 22-27. doi: http://doi.org/10.15587/2519-4798.2021.228185

(C) The Author(s) 2021

This is an open access article under the CC BY license (http://creativecommons.org/licenses/by/4.0).

\section{Introduction}

Bladder cancer (BC) is the fifth most common cancer diagnosis among the male population worldwide and the tenth most common cancer [1]. The agestandardized incidence rate worldwide (per 100,000 people/year) is 9.0 for men and 2.2 for women [1]. In Ukraine, the age-standardized incidence rate was 13.7 in men and 2.0 in women in 2018 [2]. In Europe, the highest age-standardized incidence rate is in Belgium (31 in men and 6.2 in women), and the lowest in Finland (18.1 in men and 4.3 in women) [3].

Globally, the age-standardized mortality rate (per 100,000 people/year) was 3.2 for men versus 0.9 for women in 2012 [1]. In Ukraine, the mortality rate according to the world standard was 5.1 for men and 0.5 for women in 2018 [2].

Approximately $75 \%$ of BC patients have disease confined to the mucosa (stage Ta, carcinoma in citu (CIS)) or submucosa (stage T1), and this percentage is even higher in younger patients $(<40)$ [4]. Patients with TaT1 stages and CIS have a lower risk of dying from BC compared with patients with stage T2-4 tumors [5].

The main surgical method for the treatment of non-muscle invasive bladder cancer (NMIBC) stages Ta, $\mathrm{T} 1$ is transurethral resection of the bladder wall. The purpose of this procedure in primary patients with a tumor of the bladder is the complete removal of all visible foci, as well as the implementation of certain diagnostic manipulations, for a complete and correct diagnosis.
With histological confirmation of the muscular-noninvasive nature of the tumor process, transurethral resection begins to be considered not only a diagnostic, but also a radical surgical procedure, after which indications for observation or adjuvant treatment are determined.

Adjuvant treatment should be based on the patient's prognosis. To predict both short-term and longterm risks of relapse and disease progression in individual patients, the European Organization for Research and Treatment of Cancer (EORTC) subgroup dealing with genito-urinary neoplasms has developed a scoring system and risk tables [6]. The basis for these tables is an individual database of 2596 patients diagnosed with TaT1 tumor who were randomized into seven EORTC clinical trials. Seventy-eight percent of patients received intravesical treatment, mainly chemotherapy, and about $10 \%$ received intravesical BCG therapy.

A model that predicts the risk of recurrence and progression after adjuvant intravesical BCG therapy was developed by Club Urológico Español de Tratamiento Oncológico (CUETO) (Spanish Urological Oncology Group). It is based on an analysis of 1,062 patients from four clinical trials comparing different types of intravesical BCG therapy. Patients received up to twelve instillations of uro-BCG for five to six months after transurethral resection of the bladder (TURB) [7]. Using these data, new indications for stratification into risk groups and EORTC nomograms were developed for patients receiving $\mathrm{BCG}[6]$. 
The EORTC scoring system is based on $6 \mathrm{im}$ portant clinical and pathological characteristics of the tumor: the number of tumors, tumor size, recurrence rate, stage $(\mathrm{Ta} / \mathrm{T} 1)$ and the degree of tumor differentiation (G1/G2/G3 according to the WHO 1973 classification or PUNLMP/LG/HG according to WHO classification 2004/2016), the presence of concomitant carcinoma in situ (CIS). After determining the specific gravity of each factor, a total assessment of the risk of progression and the development of tumor recurrence is carried out. Subsequently, patients are stratified into three groups of low, moderate and high risk with a subgroup of the highest risk, and, depending on the group, the tactics of adjuvant treatment are determined.

For patients with low and medium risk tumors, the prognosis of recurrence and progression is the most favourable, and with correct and timely adjuvant treatment, the rates of 3 and 5 year recurrence-free survival in these patients can reach $75 \%$ and $60 \%$, respectively [8].

High-risk T1G3 bladder tumors are considered an NMIBC category associated with a high risk of recurrence and progression after treatment with transurethral resection alone, with a recurrence rate of $50 \%$ to $70 \%$ and a tumor progression rate of $25-50 \%$ in the first 3 years after operations. More importantly, despite the provision of adjuvant intravesical therapy, patients with intermediate and high risk of NMIBC have a probability of recurrence and progression up to $52 \%$ and $20 \%$ (high risk) after five years, respectively [9].

A separate category includes patients with extremely high-risk NMIBC, as well as patients from the high-risk group in whom BCG therapy fails (BCGfailure). In such a situation, it is recommended to perform an early radical cystectomy with one of the types of urine diversion, which makes the treatment of NMIBC extremely difficult [10].

The key problems of this operation are 90-day mortality at the level of 2.3-9\% and the incidence of perioperative complications up to $80 \%$ [11]. In addition, the physical and social quality of life of patients after radical cystectomy is significantly reduced. Also, do not forget that bladder cancer is more common in older people, most of whom have poor comorbid status due to concomitant pathology [12].

Consequently, there is a clear need for alternative bladder preservation methods for NMIBC patients who are unsuitable for or refuse radical cystectomy.

Chemohyperthermia is an instrumental technique used to increase the effectiveness of intravesical chemotherapy and has shown encouraging results in the treatment of patients with NMIBC. This method involves circulation of mitomycin-C (MMC) in the bladder and simultaneous hyperthermia (HT) of the bladder wall up to $41-44{ }^{\circ} \mathrm{C}$ [13]. It is known that hyperthermia promotes direct and indirect DNA damage [14], which can enhance the anticancer immune response [15], and also increases the concentration and depth of penetration of chemotherapeutic agents into the tissues of the bladder wall and potentiates their effect on cancer cells [16].

Following current guidelines from the European Association of Urology (EAU), hyperthermic chemotherapy may be considered in patients with NMIBC in whom previous intravesical treatment has failed (BCG failure) and in those who cannot or do not want to undergo radical cystectomy, however, as an alternative BCG therapy in high-risk patients is not currently being considered [10].

The aim of this study is a preliminary analysis of oncological indicators of the effectiveness of adjuvant hyperthermic intravesical chemotherapy in patients with high-risk NMIBC compared with standard BCG therapy.

\section{Materials and methods}

A retroprospective study included 96 high-risk NMIBC patients with an average age of $65.6 \pm 11.9$ years, who received adjuvant intravesical therapy on an outpatient basis at the University Clinic of Odessa National Medical University in the period from 2013 to 2020.

When forming the groups, the following inclusion criteria were used:

- consent to conduct research;

- histologically confirmed diagnosis of high risk primary non-invasive bladder cancer (T1; G3; CIS; multiple recurrent tumor $>3 \mathrm{~cm}, \mathrm{TaG} 1 \mathrm{G} 2 / \mathrm{LG})$

- histologically confirmed diagnosis of recurrent non-muscle invasive bladder cancer of high or medium risk;

- age of patients over 18 years old;

- the state of the functional status of the patient's body according to the Karnofsky scale from 100 to 60 percent.

All studies were carried out in accordance with the Council of Europe Convention "On the Protection of Human Rights and Human Dignity in Relation to the Use of the Advances in Biology and Medicine (ETS No. 164)" dated 04.04.1997, and the Declaration of Helsinki by the World Medical Association (2008) (Protocol No. 135A meetings of the Commission on Bioethics of the Odessa National Medical University on 03/07/2019). Before the inclusion of patients in the study protocol, their personal written voluntary consents to participate in the study were obtained and all measures were taken to ensure the anonymity of patients.

Depending on the type of treatment, the patients were divided into 2 groups. Group 1 (comparison) included patients who received adjuvant therapy after TURB with the BCG vaccine (BCG therapy group; $n=50$ ), group 2 (study group) included patients (HIVEC ${ }^{\circledR}$ therapy group; $n=46$ ), who received adjuvant intravesical chemotherapy using the Combat BRS HIVEC ${ }^{\circledR}$ device for local hyperthermia.

The treatment protocol for BCG patients included the instillation of a solution of the Uro-BCG vaccine (lyophilisate containing from $2 \times 108$ to $8 \times 108$ viable BCG bacteria) at a temperature of $20-21{ }^{\circ} \mathrm{C}$, once a week into an empty bladder with a disposable urethral catheter. The patient had to withstand an exposure time of 1 hour before urinating. The main course of treatment consisted of 6 weekly procedures. The maintenance course was 1 instillation of BCG once a month for 6 months.

The treatment protocol for patients in group 2 consisted of a six-week course of Mitomycin-C instillations at a dose of $40 \mathrm{mg}$. Using an apparatus for hyperthermic chemotherapy, which extravesically heated the chemotherapy solution to $41-43{ }^{\circ} \mathrm{C}$ and recirculated it for 60 minutes at a stable pressure at a rate of 
$200 \mathrm{ml} /$ minute. All instillations were performed using the CombatBRSsystem V2.0, which was used according to the manufacturer's instructions (Combat Medical, Withamstead, UK) (Fig. 1, 2).

The study groups included only those patients who received an effective amount of instillations (at least 4).

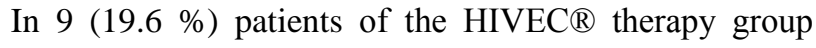
with a complicated course of the disease (the presence of a microcyst, a pronounced inflammatory reaction), $50 \mathrm{ml}$ was first injected into an empty bladder. $10 \%$ solution of dimethyl sulfoxide (Dimexide), which acted as a penetrate substance for a chemotherapy drug, after which mitomycin-C was instilled in a reduced dosage (patent for invention No. 122302, registered in the State Register of Inventions of Ukraine on 10/12/2020).

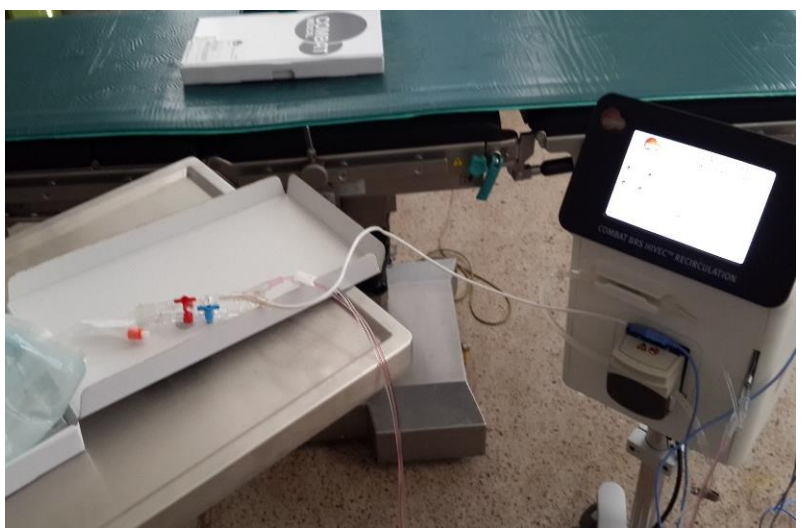

Fig. 1. Preparing the system for intravesical instillation

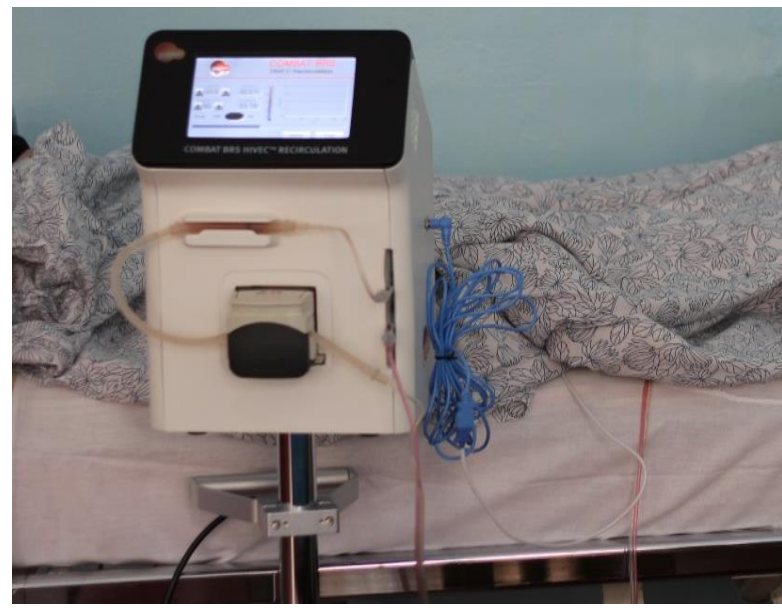

Fig. 2. Instillation of Mitomycin-C using Combat BRS HIVEC® apparatus

The characteristics of the studied groups are presented in Table 1.

Table 1 data indicate that there were no significant differences in demographic variables between patients in both groups. In addition, there was no significant imbalance in the oncological characteristics of the tumor process, such as tumor size, number of neoplasms, depth of invasion into the bladder mucosa, histological gradation, and the presence of concomitant CIS.

Table 1

\begin{tabular}{|c|c|c|}
\hline Parameter & $\begin{array}{c}\text { Group BCG } \\
N(\%) \\
\end{array}$ & $\begin{array}{c}\text { Group HIVEC® } \\
n(\%) \\
\end{array}$ \\
\hline $\begin{array}{c}\text { Sex } \\
\text { Male } \\
\text { Female }\end{array}$ & $\begin{array}{c}41(82.0) \\
9(18.0) \\
\end{array}$ & $\begin{array}{l}35(76.1) \\
11(23.9) \\
\end{array}$ \\
\hline Age & $\begin{array}{c}65.4 \pm 11.8 \\
(95 \% \mathrm{CI}: 62.12-68.42) \\
\end{array}$ & $\begin{array}{c}65.83 \pm 12.212 \\
(95 \% \mathrm{CI}: 62.24-69.30)\end{array}$ \\
\hline $\begin{array}{c}\text { Primary } \\
\text { Recurrent }\end{array}$ & $\begin{array}{l}42(84) \\
8(16) \\
\end{array}$ & $\begin{array}{l}33(71.7) \\
13(61.9) \\
\end{array}$ \\
\hline $\begin{array}{l}\mathrm{Ta} \\
\mathrm{T} 1\end{array}$ & $\begin{array}{c}5(10) \\
45(90)\end{array}$ & $\begin{array}{c}3(6.5) \\
43(93.5) \\
\end{array}$ \\
\hline $\begin{array}{c}\text { Number of tumors } \\
1 \\
2-7 \\
\end{array}$ & $\begin{array}{l}26(52) \\
24(48)\end{array}$ & $\begin{array}{l}27(58.7) \\
19(41.3) \\
\end{array}$ \\
\hline $\begin{array}{c}\text { Tumour diameter } \\
<3 \\
>=3\end{array}$ & $\begin{array}{l}25(50) \\
25(50)\end{array}$ & $\begin{array}{l}22(47.8) \\
24(52.2)\end{array}$ \\
\hline CIS & $10(20)$ & $8(17.4)$ \\
\hline $\begin{array}{l}\text { Low grade } \\
\text { High grade }\end{array}$ & $\begin{array}{l}30(60) \\
20(40)\end{array}$ & $\begin{array}{l}21(45.7) \\
25(54.3)\end{array}$ \\
\hline
\end{tabular}

Every three months, all patients underwent cystoscopy and cytological analysis of urine for atypical cells was performed, with endoscopic suspicion of recurrence or positive cytological examination, a bladder biopsy was performed. Spiral computed tomography of the urinary tract was performed once a year. The primary outcome was disease-free survival (DFS), defined as the time from initiation of the first intravesical chemohyperthermia procedure to disease recurrence and / or progression. Relapse was defined as a histologically confirmed diagnosis of urothelial carcinoma, while progression was defined as a histologically or radiologically confirmed diagnosis of muscle-invasive bladder cancer or metastatic disease. 
Comparative analysis of the studied parameters in the comparison group was carried out according to the $\chi^{2}$ method and using the t-test for independent samples. To obtain DFS estimates, the Kaplan-Meier method was used, the indicators were compared between using a logrank test. Patients were censored by date of recurrence or disease progression, or by date of last cystoscopy. IBM SPSS Statistics for Windows (version 26.0) was used as a program for calculating statistical indicators. A $p$ value $<0.05$ was considered an indicator of statistical significance.

\section{Research results}

From the analysis were excluded 7 patients $(2$ in the HIVEC ${ }^{\circledR}$ group and 5 in the BCG group) who discontinued treatment in the first and second cycles of intravesical therapy. Six-week cycles were not completely completed due to the developed side effects of treatment in $13(26 \%)$ patients from the BCG therapy group (7 received 4 sessions of instillation, $6-5$ sessions), in the chemohyperthermia group $4(8.7 \%)$ did not complete a complete course of therapy (1 patient received 4 sessions of instillation, three -5 sessions $)(p=0.005)$. In $18(36 \%)$ of 50 patients of the first group and $8(17.4 \%)$ of 35 patients of group 2, the schedule of instillations was once shifted by a week due to moderate local toxicity, twice in $8(16 \%)$ and $2(4.3 \%)(\mathrm{p}<0.05)$.

The median follow-up period was 23 months (range 4-36), the median follow-up period between patients in the first and second groups was 25.8 versus
20.0 months, respectively $(p=0.002)$. Tumor recurrence was reported in 19 of the patients receiving intravesical BCG therapy and in 8 patients receiving intravesical hyperthermic chemotherapy. The recurrence rate was significantly different between the two groups (38\% versus $17.4 \%, \mathrm{p}=0.025$ ) (Table 2).

Table 2

Immediate outcomes of adjuvant treatment for high-risk NMIBC patients

\begin{tabular}{|l|c|c|}
\hline \multicolumn{1}{|c|}{ Occasion } & $\begin{array}{c}\text { Group BCG } \\
n(\%)\end{array}$ & $\begin{array}{c}\text { Group HIVEC } \mathbb{} \\
n(\%)\end{array}$ \\
\hline Relapse & $19(38)$ & $8(17,4)^{*}$ \\
Yes & $31(62)$ & $38(82,6)$ \\
No & $26.5(95 \mathrm{CI}:$ & $26,9(95 \mathrm{CI}:$ \\
\hline Average rate of & $22,6-29,7)$ & $25,0-28,8)^{* *}$ \\
DFS & \multicolumn{2}{|c|}{ Note: $*$ - index $p=0.025 ; *$-index $p=0.029$}
\end{tabular}

An analysis of disease-free survival (DFS) for the two adjuvant treatment strategies is shown in Fig. 3. The incidence of DFS in patients receiving intravesical hyperthermic chemotherapy was significantly higher than in patients receiving intravesical BCG therapy (log-rank test: $p=0.029$ ).

Overall survival (OS) during follow-up did not differ statistically and amounted to $82 \%$ in the BCG therapy group and $89 \%$ in the HIVEC ${ }^{\circledR}$ therapy group (log-rank test result: $p=0.785$ ) (Fig. 4).

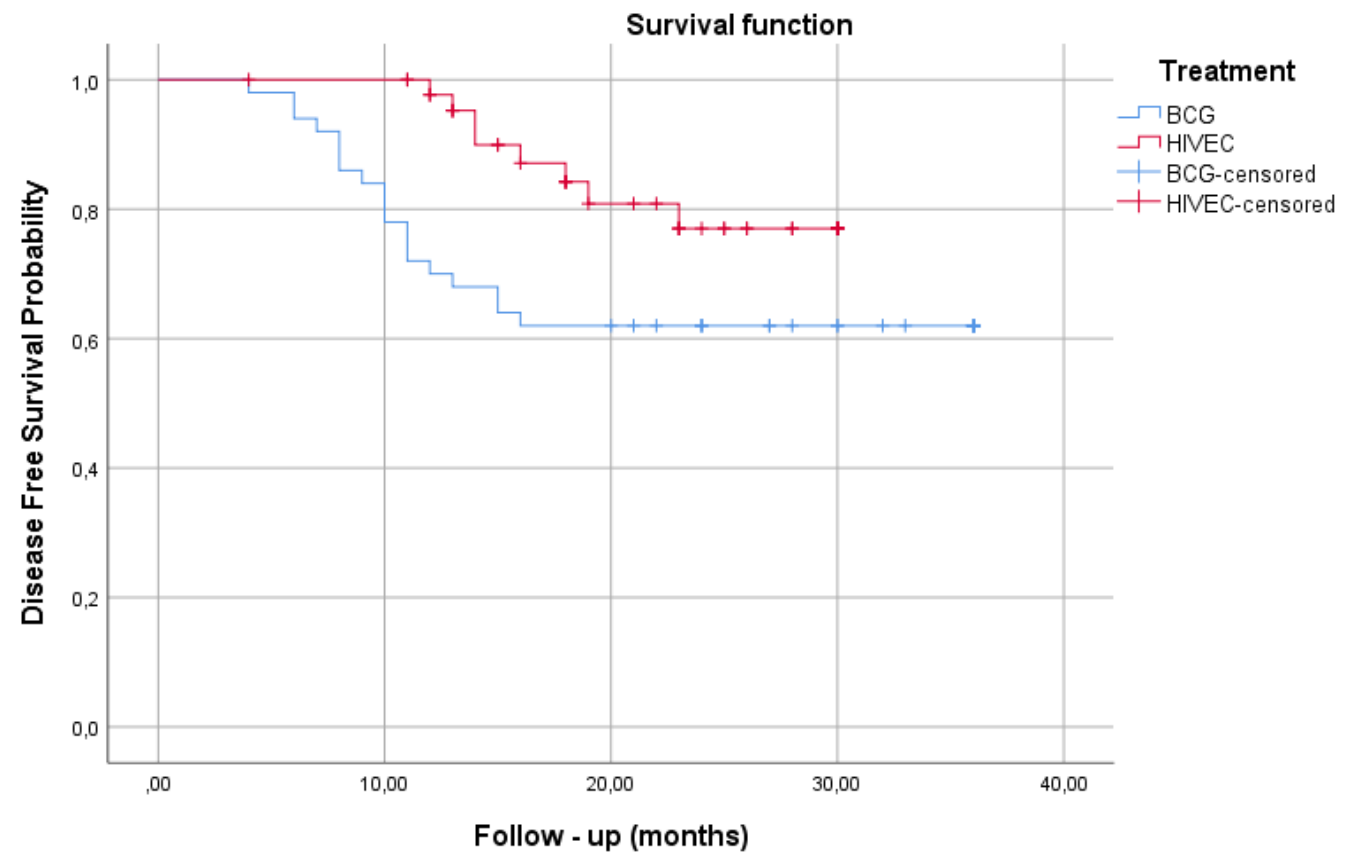

Fig. 3. Kaplan-Meier curves of DFS indicators of the studied groups 


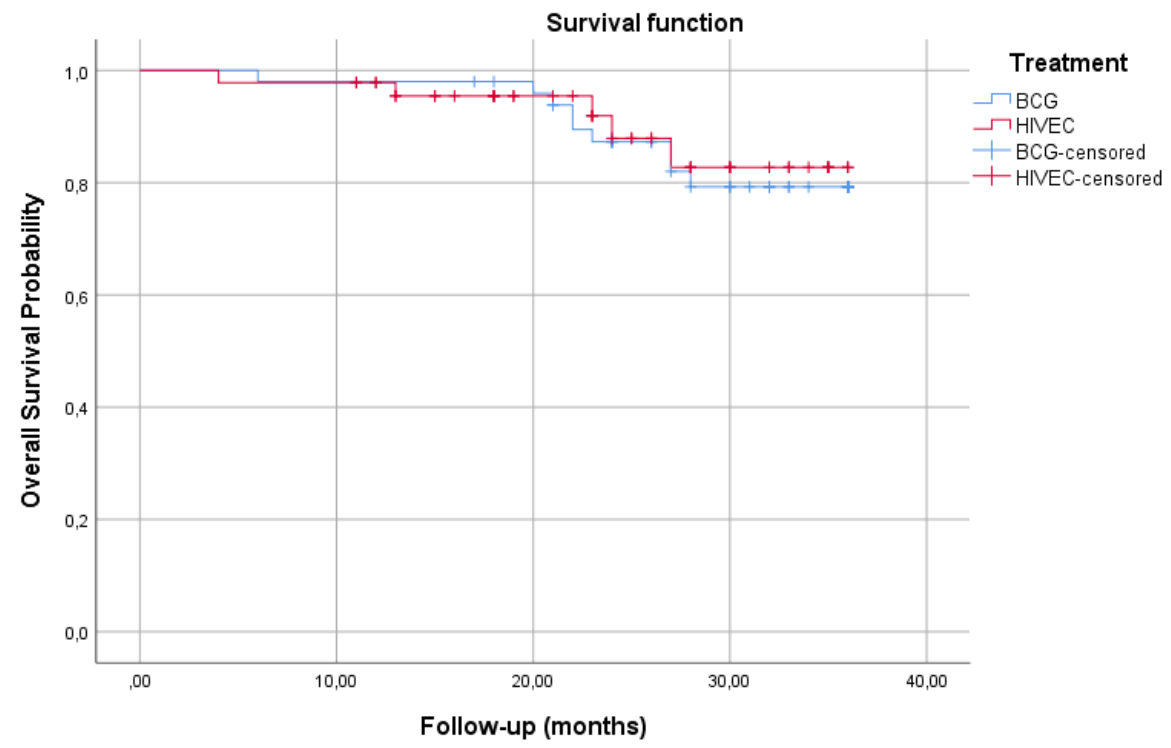

Fig. 4. Kaplan-Meier curves of OS indicators of the studied groups

\section{Discussion of research results}

Preliminary results of adjuvant treatment of patients with high-risk non-muscle invasive bladder cancer are presented. The study of drug strategies in this cohort of patients is still actively pursued by urologists, as these patients have a high risk of recurrence and progression. Currently, there are two main tactics: early radical cystectomy or transurethral resection (TURB) followed by adjuvant intravesical uro-BCG therapy. Radical cystectomy for non-muscle invasive bladder cancer may not be suitable for all categories of patients (for reasons of age, comorbidity, rejection). Adjuvant intravesical BCG therapy is currently considered the gold standard for organsparing treatment of patients with high-risk NMIBC, but the toxicity of this type of therapy does not always allow for a full 6-week induction course, and it is also quite difficult to control the exposure time of the drug [17]. All this makes us look for opportunities to improve methods of intravesical therapy by developing new medicinal agents and techniques that enhance their effectiveness.

One of the possible methods for increasing the oncological effectiveness of intravesical chemotherapy is apparatus hyperthermia. In this study, the hyperthermic effect was achieved by increasing the temperature of the Mitomycin-C solution itself.

At the same time, this technique showed satisfactory patient tolerance during the treatment cycle $-91.4 \%$ of patients in the HIVEC $\AA$ therapy group completed it in comparison with $74 \%$ in the BCG therapy group $(p=0.005)$.

The relapse rate during follow-up was significantly different between the two groups (38\% versus $17.4 \%$; $p=0.025$ ), while the mean relapse-free survival rate in HIVEC® patients was also higher than in BCG-treated patients (respectively 26.9 (95CI: 25.0-28.8) and 26.5 (95CI: 22.6-29.7), the result of the log-rank test: $P=0.029)$.

104 patients were included in the only randomized controlled study of the use of chemohyperthermia in patients with high-risk NMIBC in whom BCG therapy was ineffective (BCG-failure) (48 in the group receiving Mi-
tomycin-C in combination with microwave-induced hyperthermia; 56 in control). The median follow-up of 31 patients without recurrence or progression was 36 months. The study group achieved $35 \%$ disease-free survival (DFS) after 2 years compared with $41 \%$ of the control group (who received $\mathrm{BCG}, \mathrm{MMC}$, or instrumental drug administration at the discretion of the investigator) [18].

Another study of microwave-induced chemohyperthermia enrolled 299 patients with moderate to highrisk NMIBC recurrence, of which $255(85.3 \%)$ had previously received BCG therapy (217 BCG-failure patients). Patients were stratified into groups according to the presence of CIS, as well as according to the tactics of treatment - adjuvant or ablative. According to the analysis results, 2-year relapse-free survival was $57.5 \%$ (95\% CI: 48.9-66.1), in the group with concomitant CIS $66.5 \%$ (95\% CI: 54.3-78.7), 5-year relapse-free survival was $37.2 \%$ (95\% CI: $28.4-46.0)$, in the group with concomitant CIS $40.3 \%$ (95 \% CI: 25.2-55.4), 5-year overall and carcinoma-specific survival (CSS) were $72.3 \%$ (66.4-87.2) and 86.6\% (81.7-91.5), respectively [19].

Study limitations. Given the retrospective nature of the study, the BCG therapy group began to form before the introduction of the method of hyperthermic chemotherapy into practice; this is associated with the differences in the medians of observation of patients in both groups. In the future, with equalization of the median follow-up time, an increase in the number of relapses and a decrease in the DFS index in the study group of hyperthermic intravesical chemotherapy can be expected.

Prospects for further research. The study of the histological and immunohistochemical characteristics of primary and recurrent tumors will create an algorithm for more effective adjuvant treatment of patients with NMIBC, and will expand the number of organpreserving techniques.

\section{Conclusions}

The method of hyperthermic intravesical chemotherapy, in comparison with the standard method of adjuvant conservative therapy of patients with high- 
risk NMIBC, upon preliminary analysis of the results, significantly increases the 2-year disease-free survival $(82.6 \%$ versus $62 \%, p=0.025)$. Taking into account the better tolerability of this method of intravesical treatment, its application in the future will increase the frequency of organ-preserving treatment of patients with primary and recurrent muscular-non-invasive bladder tumors.

\section{Conflict of interests}

The authors declare that they have no conflicts of interest.

\section{References}

1. Sung, H., Ferlay, J., Siegel, R. L., Laversanne, M., Soerjomataram, I., Jemal, A., Bray, F. (2021). Global cancer statistics 2020: GLOBOCAN estimates of incidence and mortality worldwide for 36 cancers in 185 countries. CA: A Cancer Journal for Clinicians. doi: http://doi.org/10.3322/caac. 21660

2. Fedorenko, Z. P., Hulak, L. O., Mykhailovych, Yu. Y., Horokh, Ye. L., Ryzhov, A. Yu., Sumkina, O. V., Kutsenko, L. B. (2020). Rak v Ukraini, 2018-2019. Zakhvoriuvanist, smertnist, pokaznyky diialnosti onkolohichnoi sluzhby. Biuleten Natsionalnoho kantser-reiestru Ukrainy, 21, 101.

3. Burger, M., Catto, J. W. F., Dalbagni, G., Grossman, H. B., Herr, H., Karakiewicz, P. et. al. (2013). Epidemiology and Risk Factors of Urothelial Bladder Cancer. European Urology, 63 (2), 234-241. doi: http://doi.org/10.1016/j.eururo.2012.07.033

4. Chavan, S., Bray, F., Lortet-Tieulent, J., Goodman, M., Jemal, A. (2014). International Variations in Bladder Cancer Incidence and Mortality. European Urology, 66 (1), 59-73. doi: http://doi.org/10.1016/j.eururo.2013.10.001

5. Compérat, E., Larré, S., Roupret, M., Neuzillet, Y., Pignot, G., Quintens, H. et. al. (2015). Clinicopathological characteristics of urothelial bladder cancer in patients less than 40 years old. Virchows Archiv, 466 (5), 589-594. doi: http://doi.org/10.1007/s00428-015-1739-2

6. Cambier, S., Sylvester, R. J., Collette, L., Gontero, P., Brausi, M. A., van Andel, G. et. al. (2016). EORTC Nomograms and Risk Groups for Predicting Recurrence, Progression, and Disease-specific and Overall Survival in Non-Muscle-invasive Stage Ta-T1 Urothelial Bladder Cancer Patients Treated with 1-3 Years of Maintenance Bacillus Calmette-Guérin. European Urology, 69 (1), 60-69. doi: http://doi.org/10.1016/j.eururo.2016.01.055

7. Xylinas, E., Kent, M., Kluth, L., Pycha, A., Comploj, E., Svatek, R. S. et. al. (2013). Accuracy of the EORTC risk tables and of the CUETO scoring model to predict outcomes in non-muscle-invasive urothelial carcinoma of the bladder. British Journal of Cancer, 109 (6), 1460-1466. doi: http://doi.org/10.1038/bjc.2013.372

8. Sylvester, R. J., Oosterlinck, W., Holmang, S., Sydes, M. R., Birtle, A., Gudjonsson, S. et. al. (2016). Systematic Review and Individual Patient Data Meta-analysis of Randomized Trials Comparing a Single Immediate Instillation of Chemotherapy After Transurethral Resection with Transurethral Resection Alone in Patients with Stage pTa-pT1 Urothelial Carcinoma of the Bladder: Which Patients Benefit from the Instillation? European Urology, 69 (2), 231-244. doi: http://doi.org/10.1016/j.eururo.2015.05.050

9. Malmström, P.-U., Sylvester, R. J., Crawford, D. E., Friedrich, M., Krege, S., Rintala, E. et. al. (2009). An Individual Patient Data Meta-Analysis of the Long-Term Outcome of Randomised Studies Comparing Intravesical Mitomycin C versus Bacillus Calmette-Guérin for Non-Muscle-Invasive Bladder Cancer. European Urology, 56 (2), 247-256. doi: http://doi.org/10.1016/j.eururo.2009.04.038

10. Babjuk, M., Burger, M., Compérat, E., Gontero, P., Mostafid, A. H., Palou, J. et. al. (2020). EAU Guideline Non-MuscleInvasive Bladder Cancer. Proceedings of the EAU Annual Congress Amsterdam 2020. Virtual Congress. Available at: https://uroweb.org/guideline/non-muscle-invasive-bladder-cancer/ Last accessed: 26.08.2020

11. Hautmann, R. E., de Petriconi, R. C., Volkmer, B. G. (2010). Lessons Learned From 1,000 Neobladders: The 90-Day Complication Rate. Journal of Urology, 184 (3), 990-994. doi: http://doi.org/10.1016/j.juro.2010.05.037

12. Parker, W. P., Smelser, W., Lee, E. K., Habermann, E. B., Thapa, P., Zaid, H. B. et. al. (2018). Utilization and Outcomes of Radical Cystectomy for High-grade Non-muscle-invasive Bladder Cancer in Elderly Patients. Clinical Genitourinary Cancer, 16 (1), e79-e97. doi: http://doi.org/10.1016/j.clgc.2017.07.011

13. Van Valenberg, H.; Colombo, R.; Witjes, F. Intravesical radiofrequency-induced hyperthermia combined with chemotherapy for non-muscle-invasive bladder cancer. Int. J. Hyperth. 2016, 32, 351-362. https://doi.org/10.3109/02656736.2016.1140232

14. Mantso, T., Goussetis, G., Franco, R., Botaitis, S., Pappa, A., Panayiotidis, M. (2016). Effects of hyperthermia as a mitigation strategy in DNA damage-based cancer therapies. Seminars in Cancer Biology, 37-38, 96-105. doi: http://doi.org/10.1016/j.semcancer.2016.03.004

15. Tan, W. S., Kelly, J. D. (2018). Intravesical device-assisted therapies for non-muscle-invasive bladder cancer. Nature Reviews Urology, 15 (11), 667-685. doi: http://doi.org/10.1038/s41585-018-0092-z

16. Van Valenberg, F. J. P., van der Heijden, A. G., Lammers, R. J. M., Falke, J., Arends, T. J. H., Oosterwijk, E., Witjes, J. A. (2017). Intravesical radiofrequency induced hyperthermia enhances mitomycin C accumulation in tumour tissue. International Journal of Hyperthermia, 34 (7), 988-993. doi: http://doi.org/10.1080/02656736.2017.1406618

17. Kostyev, F. I., Bondar, O. V., Chystiakov, R. S., Lysenko, V. V. (2020). Impact of adjuvant intrabladder therapy on quality of life in patients, suffering musculo-noninvasive cancer of the bladder. Klinicheskaia Khirurgiia, 87 (7-8), 47-52. doi: http://doi.org/10.26779/2522-1396.2020.7-8.47

18. Tan, W. S., Panchal, A., Buckley, L., Devall, A. J., Loubière, L. S., Pope, A. M. et. al. (2019). Radiofrequency-induced Thermochemotherapy Effect Versus a Second Course of Bacillus Calmette-Guérin or Institutional Standard in Patients with Recurrence of Nonmuscle-invasive Bladder Cancer Following Induction or Maintenance Bacillus Calmette-Guérin Therapy (HYMN): A Phase III, Open-label, Randomised Controlled Trial. European Urology, 75 (1), 63-71. doi: http://doi.org/10.1016/j.eururo.2019.01.030

19. Brummelhuis, I. S. G., Wimper, Y., Witjes-van Os, H. G. J. M., Arends, T. J. H., van der Heijden, A. G., Witjes, J. A. (2021). Long-Term Experience with Radiofrequency-Induced Hyperthermia Combined with Intravesical Chemotherapy for NonMuscle Invasive Bladder Cancer. Cancers, 13 (3), 377. doi: http://doi.org/10.3390/cancers13030377

Received date 03.12.2020

Accepted date 04.01.2021

Published date 31.03.2021

Roman Chystiakov, Assistant, Department of Radiation Diagnostic, Therapy and Oncology, Odessa National Medical University, Valihovskiy lane, 2, Odessa, Ukraine, 65082

E-mail: romanchystiakov@gmail.com 